\title{
Effects of dietary trans-9 octadecenoic acid, trans-11 vaccenic acid and cis-9, trans-11 conjugated linoleic acid in mice
}

\author{
TAO WANG ${ }^{1,2^{*}}$, JI-NA LIM ${ }^{3 *}$, JAE-SUNG LEE $^{3}$, SANG-BUM LEE $^{3}$, JIN-HEE HWANG $^{3}$, U-SUK JUNG ${ }^{3}$, \\ MIN-JEONG KIM ${ }^{3}$, DAE-YOUN HWANG ${ }^{4}$, SANG-RAK LEE ${ }^{3}$, SANG-GUN ROH ${ }^{5}$ and HONG-GU LEE ${ }^{3}$ \\ ${ }^{1}$ Department of Animal Nutrition and Feed Science, College of Animal Science and Technology; \\ ${ }^{2}$ Key Laboratory of Animal Nutrition and Feed Science, Jilin Agricultural University, Changchun, Jilin 130118, P.R. China; \\ ${ }^{3}$ Department of Animal Science and Technology, College of Animal Bioscience and Technology, Konkuk University, Seoul 143-701; \\ ${ }^{4}$ Department of Biomaterials Science, College of Natural Resources and Life Science, Pusan National University, \\ Miryang, Gyeongnam 627-706, Republic of Korea; ${ }^{5} \mathrm{Lab}$ of Animal Physiology, Graduate School of Agriculture Science, \\ Tohoku University, Sendai, Miyagi 988-8555, Japan
}

Received July 22, 2014; Accepted April 10, 2015

DOI: $10.3892 / \mathrm{mmr} .2015 .3767$

\begin{abstract}
The aim of the present study was to investigate the effects of dietary trans fatty acids in mice. Following the administration of a $0.5 / 100 \mathrm{~g}$ diet of trans- 9 octadecenoic acid (EA), trans-11 vaccenic acid (TVA) or cis-9, trans-11 conjugated linoleic acid (CLA) for 4 weeks, the body weights and the weights of the liver, testis and mediastinal adipose tissue (MAT) of the animals gradually decreased $(\mathrm{P}<0.05)$. The EA group exhibited the lowest levels of magnesium and triglycerides $(\mathrm{P}<0.05)$. CLA increased villus length $(\mathrm{P}<0.05)$, while EA and TVA decreased villus length $(\mathrm{P}<0.05)$. The TVA group exhibited the lowest levels of low-density lipoprotein and tumor necrosis factor- $\alpha(\mathrm{P}<0.05)$. Taken together, EA, TVA and CLA affected the physiological conditions of mice differently. The potential effects of three well-known fatty acids, including trans-9 octadecenoic acid (EA), trans-11 vaccenic acid (TVA) and cis-9, trans-11 conjugated linoleic acid (CLA), in animals or humans remain to be elucidated. Therefore, in the present study, 32 animals were randomly divided into four groups and administered a 0.5/100 g diet of EA, TVA or CLA for 4 weeks. The results demonstrated that the body weights and the weights of the liver, testis and mediastinal adipose tissue (MAT) of the animals gradually decreased $(\mathrm{P}<0.05)$. Blood was collected individually
\end{abstract}

Correspondence to: Professor Hong-Gu Lee, Department of Animal Science and Technology, College of Animal Bioscience and Technology, Konkuk University, 120 Neungdong-ro, Seoul 143-701, Republic of Korea

E-mail: hglee66@konkuk.ac.kr

*Contributed equally

Key words: cis-9, trans-11 conjugated linoleic acid, physiological conditions, trans- 9 octadecenoic acid, trans-11 vaccenic acid via the external jugular veins and the EA group exhibited the lowest levels of magnesium and triglycerides $(\mathrm{P}<0.05)$. CLA increased villus length $(\mathrm{P}<0.05)$, while EA and TVA decreased villus length $(\mathrm{P}<0.05)$. The TVA group exhibited the lowest levels of low-density lipoprotein and tumor necrosis factor- $\alpha(\mathrm{P}<0.05)$. Taken together, EA, TVA and CLA affected the physiological conditions of mice differently and these may further our understanding of the various effects of these fatty acids on animals and humans.

\section{Introduction}

Trans-9 octadecenoic acid (EA) is produced during catalytic partial hydrogenation in the production of hardened fats and during deodorization of commodity oils, and it is also present in dairy fats (1). Trans-11 vaccenic acid (TVA) is a known positional and geometric isomer of oleic acid and is the dietary precursor of cis-9, trans-11 conjugated linoleic acid (CLA) $(2,3)$. This fatty acid is an intermediate of ruminal biohydrogenation, which has received significant attention due to its health benefits, associated with and without CLA (3-5). Cis-9, trans-11 CLA is one of a number of positional and geometric isomers of linoleic acid (6). This CLA isomer exerts several isomer-specific effects, including anti-atherosclerotic, anti-inflammatory and anticarcinogenic effects, and immune system modulation (3). These three fatty acids have received attention due to their specific effects and their wide distribution in human food.

In low-density lipoprotein (LDL) receptor-deficient mice, a diet of EA-rich hydrogenated vegetable shortening for 14 weeks led to increased atherosclerosis, compared with butter rich in TVA (7). When TVA-/CLA-enriched butter was fed to rodents, the serum levels of cholesterol and triacylglycerol, and the extent of atherosclerosis were reduced compared with those fed with regular butter $(8,9)$. In rabbits, the ratio of atherogenic to antiatherogenic lipoproteins, very-low-density lipoprotein (VLDL)+LDL to high-density lipoprotein (HDL), was observed to be significantly lower 
in animals fed TVA/CLA-enriched butter, compared with those fed trans-10-C18:1-enriched group (9). In addition, milk fat-rich cis-9, trans-11 CLA has been demonstrated to reduce the atherogenic process in hyperlipidemic hamsters (10), and bovine milk fat, enriched with conjugated linoleic and vaccenic acids, attenuates allergic airway disease in mice (11). The above findings were obtained using butter, which was naturally enriched with EA, TVA or cis-9, trans-11 CLA, or all three, however, the pure form of these fatty acids was not used, and further investigations are required to confirm these findings. Therefore, in the present study, system-wide analyses were performed to assess the potential effects of pure EA, TVA or cis-9, trans-11 CLA in mice.

\section{Materials and methods}

Subjects. All experiments involving animals were approved by the Animal Care and Use Committee of Pusan National University (Miryang, Korea; PNU-2012-0056). Male, 6-week-old, Institute of Cancer Research (ICR) mice (Japan SLC Inc., Shizuoka, Japan) were acclimated for 7 days prior to assessment of health status. The study was approved by the ethics committee of Pusan National University (Miryang, Korea). The mice were individually housed with sawdust bedding and were maintained in environmentally controlled rooms (ambient temperature, $22 \pm 2^{\circ} \mathrm{C}$; relative humidity, $50 \pm 10 \%$ ) under a 12/12 h light-dark cycle (lights on 8:00 am-8:00 pm). Food and water were available ad libitum. The repeated dose (4 weeks) investigation was performed in 32 mice, which were divided into four groups of eight animals: Control (7\% soybean oil), EA (6.5\% soybean oil $+0.5 \%$ EA), TVA $(6.5 \%$ soybean oil $+0.5 \%$ TVA) and CLA (6.5\% soybean oil $+0.5 \%$ cis -9 , trans-11 CLA), as shown in Table I. The dosages of each fatty acid were selected on the basis of the worldwide consumption report (12). The fatty acids were obtained from Matreya LLC (Pleasant Gap, PA, USA). The experimental diets were manufactured by Feedlab (Kyung-ki, Seoul, Korea), and no deterioration of the fatty acids in the diets was observed following storage for 4 weeks (data not shown).

The total fatty acid compositions of the diets were analyzed and are described in Table I. Briefly, the total lipids were extracted from $4 \mathrm{~g}$ of feed using $20 \mathrm{ml}$ chloroform/methanol (2:1; v/v; Burdick \& Jackson, Muskegon, MI, USA). The extracted lipids were subsequently converted into fatty acid methyl esters using $14 \%(\mathrm{w} / \mathrm{v})$ boron trifluoride-methanol (cat. no. B1252; Sigma-Aldrich, St. Louis, MO, USA), according to a previously published method (13). The fatty acid methyl esters were then subjected to gas chromatography (Agilent 7890A GC system; Agilent Technologies, Inc., Santa Clara, CA, USA) using a system equipped with a 7863 series auto-sampler, 7683B series injector and flame ionization detector. An SP ${ }^{\text {тм }}-2560$ fused silica capillary column (L x I.D. $100 \mathrm{~m} \times 0.25 \mathrm{~mm}$, df $0.20 \mu \mathrm{m}$ ) was used (Supelco Inc., Bellefonte, PA, USA) to analyze the fatty acid compositions of the samples. The peaks were routinely identified by comparing the duration of retention with those of the standards, including Supelco 37 Component Fatty acid methyl esters mix (47885-U), trans-11-octadecenoicmethyl ester (46905-U; Sigma-Aldrich) and cis-9, trans-11 CLA
(1255; Matreya LLC, Pleasant Gap, PA, USA). The percentages of the individual fatty acids were calculated as the ratio of each area compared with that of the total identified fatty acids, as described previously (14). The four experimental diets contained a similar concentration of fatty acids. The concentrations of the three assessed fatty acids decreased to $0.25 \%$ from an initial concentration of $0.5 \%$, which may have been due to losses acquired during production (Table I). The body weights and food intake of each mice were measured every 2 days. In addition, the general appearance, behavior and signs of morbidity and mortality were observed every 2 days throughout the experiment.

Blood sampling and blood metabolite analysis. Following 4 weeks of dietary intervention, the mice were sacrificed by $\mathrm{CO}_{2}$ asphyxiation and blood samples were collected $(5 \mathrm{ml})$ from the external jugular veins. The blood was centrifuged at $400 \mathrm{x} \mathrm{g}$ at $4^{\circ} \mathrm{C}$ for $15 \mathrm{~min}$ and the supernatant was collected and stored at $-80^{\circ} \mathrm{C}$ until subsequent analysis.

The blood metabolites were analyzed using a Toshiba Accute Biochemical Analyzer-TBA-40FR (Toshiba Medical Instruments, Tochigi-ken, Japan), according to the manufacturer's instructions. A total of 10 metabolites, including blood urea nitrogen, calcium, magnesium, triglyceride, glucose, non-esterified fatty acid, albumin, total cholesterol, LDL cholesterol and HDL cholesterol were analyzed. All the reagents required for this procedure were purchased from Wako Pure Chemical Industries, Ltd. (Chuo-ku, Osaka, Japan).

Anatomical pathology. Samples of the small intestine and liver ( $\mathrm{n}=80$ /group) were collected and fixed in formaldehyde solution (Sigma-Aldrich). The samples for histopathological examination were processed, embedded in paraffin (Sigma-Aldrich), cut to a thickness of $\sim 2-4 \mu \mathrm{m}$ and stained using hematoxylin and eosin (Sigma-Aldrich). The length of the villi were then quantified ( $n=80 /$ group) by measuring the distance between the crypt necks.

Western blotting. The relative protein quantities of cholesterol $7 \alpha$-hydroxylase (CYP7 $\alpha 1)$ in the liver were determined using western blotting. Briefly, the proteins (15 $\mu \mathrm{g}$ protein/lane) were separated using $10 \%$ sodium dodecyl sulfate polyacrylamide gel electrophoresis (SDS-PAGE; Bio-Rad Laboratories, Inc., Hercules, CA, USA) and were subsequently transferred onto a polyvinylidenedifluoride membrane (GE Healthcare Life Sciences., Piscataway, NJ, USA). The membrane was blocked with phosphate-buffered saline (PBS)-T, containing 10\% 10X PBS (pH 7.6), 0.2 M Tris base, $1.37 \mathrm{M} \mathrm{NaCl}$ and $10 \%$ Tween-20, with 5\% skim milk (BD Biosciences, San Jose, CA, USA), for $1 \mathrm{~h}$ at room temperature. The membrane was incubated overnight with specific primary antibodies against rabbit monoclonal $\beta$-actin (1:1,000; cat. no. ab32575; Abcam, Cambridge, MA, USA) and mouse monoclonal CYP7 $\alpha 1$ $\left(1: 1,000\right.$; cat. no. ab77157; Abcam) at $4^{\circ} \mathrm{C}$. Following washing three times with TBS-T, the membrane was incubated with horseradish peroxidase (HRP)-conjugated goat anti-rabbit immunoglobulin G (1:1,000; cat. no. ab6721; Abcam) for $30 \mathrm{~min}$ at room temperature. The membrane was then washed with TBS-T and antibody binding was visualized using an 
Table I. Composition of the experimental diets.

\begin{tabular}{|c|c|c|c|c|}
\hline Ingredient & Control & EA & TVA & CLA \\
\hline Corn starch & 39.8 & 39.8 & 39.8 & 39.8 \\
\hline Casein-vitamin free & 20.0 & 20.0 & 20.0 & 20.0 \\
\hline Maltodextrin & 13.2 & 13.2 & 13.2 & 13.2 \\
\hline Sucrose & 10.0 & 10.0 & 10.0 & 10.0 \\
\hline Soybean oil & 7.0 & 6.5 & 6.5 & 6.5 \\
\hline Powdered cellulose & 5.0 & 5.0 & 5.0 & 5.0 \\
\hline AIN 93G mineral mix & 3.5 & 3.5 & 3.5 & 3.5 \\
\hline AIN 93G vitamin mix & 1.0 & 1.0 & 1.0 & 1.0 \\
\hline L-cystine & 0.3 & 0.3 & 0.3 & 0.3 \\
\hline Choline bitartrate & 0.25 & 0.25 & 0.25 & 0.25 \\
\hline t-Butyl-hydroquinone & 0.0014 & 0.0014 & 0.0014 & 0.0014 \\
\hline Pure fatty acid & 0.0 & 0.5 & 0.5 & 0.5 \\
\hline Total & 100 & 100 & 100 & 100 \\
\hline \multicolumn{5}{|l|}{ Proximate composition } \\
\hline Crude fat $(\%)$ & $6.4 \pm 0.0$ & $6.9 \pm 0.0$ & $6.9 \pm 0.0$ & $7.1 \pm 0.0$ \\
\hline Crude protein (\%) & $15.9 \pm 0.0$ & $15.8 \pm 0.1$ & $16.2 \pm 0.1$ & $14.4 \pm 0.1$ \\
\hline Calorie (kcal/g) & 4.319 & 4.396 & 4.379 & 4.355 \\
\hline \multicolumn{5}{|c|}{ Fatty acid composition (\%) } \\
\hline EA & ND & $2.7 \pm 0.02^{\mathrm{a}}$ & ND & ND \\
\hline TVA & ND & ND & $2.8 \pm 0.27$ & ND \\
\hline CLA & ND & ND & ND & $2.4 \pm 0.03$ \\
\hline Oleic acid & $5.3 \pm 0.05$ & $4.5 \pm 0.14$ & $4.8 \pm 0.28$ & $4.7 \pm 0.07$ \\
\hline Linoleic acid & $12.3 \pm 0.18$ & $10.4 \pm 0.32$ & $11.1 \pm 0.51$ & $10.2 \pm 0.15$ \\
\hline Linolenic acid & $1.6 \pm 0.02$ & $1.4 \pm 0.04$ & $1.5 \pm 0.07$ & $1.4 \pm 0.02$ \\
\hline
\end{tabular}

ND, not detectable. ${ }^{a}$ Values were expressed as mean \pm standard error of the mean $(n=3)$. EA, octadecenoic acid; TVA, trans-11 vaccenic acid; CLA, cis-9, trans-11 conjugated linoleic acid.

enhanced chemiluminescence system and detection kit (GE Healthcare Life Sciences, Pittsburgh, PA, USA), according to the manufacturer's instructions. The membrane was then exposed to X-ray film (Fujifilm Corporation, Minato-ku, Tokyo, Japan) for $1 \mathrm{~min}(\beta$-actin) or $3 \mathrm{~min}(\mathrm{CYP} 7 \alpha 1)$. The film was scanned and bands were quantified using the Image $\mathrm{J}^{\circledR} 1.43$ software (NIH, Bethesda, MD, USA). The protein levels were normalized against that of $\beta$-actin on the same membrane.

Cytokine production. The concentrations of tumor necrosis factor- $\alpha$ (TNF- $\alpha)$ in the plasma were determined using an ELISA kit (cat. no. 88-7324; eBioscience, Inc., San Diego, CA, USA) at the end of the experimental period, according to the manufacturer's instructions. The absorbance was then measured at $450 \mathrm{~nm}$ using a Multiskan EX Microplate Reader (Thermo Fisher Scientific Inc., Waltham, MA, USA).

Statistical analysis. All data are expressed as the mean \pm standard error of the mean and were analyzed using one-way analysis of variance with SPSS 11.5 software (SPSS Inc., Chicago, IL, USA). $\mathrm{P}<0.05$ was considered to indicate a statistically significant difference.

\section{Results and Discussion}

Changes in daily food intake, body weight and organ parameters. Changes in the general condition and external appearance of the rodents were observed in mice fed with EA or TVA. The animals of these two groups were small and weak in appearance, and their fur, eyes and oral cavity conditions were poor (data not shown). Although all the mice had a similar daily food intake (Fig. 1A), the body weights of the animals in the EA, TVA and CLA groups were all gradually decreased following 10 days administration of the assessed fatty acids (Fig. 1B). However, no significant change was observed in the lengths of the intestine or colon (Fig. 1C). In addition, the weights of the mediastinal adipose tissue (MAT) of the animals in these three groups were decreased significantly, compared with the control group $(\mathrm{P}<0.05$; Fig. 1C).

A previous study reported that TVA exerted no effect on either the body weight or food intake in obese or lean rats during experimental periods (15). In another previous study, no significant differences in body weight or body weight gain were observed between groups treated with enriched dairy fat compared with the control throughout the experimental period (16). However, in the present study, the body weight 
Table II. Ratio of organ/body weight (\%) in mice following repeated oral administration of EA, TVA or CLA for 4 weeks.

\begin{tabular}{|c|c|c|c|c|}
\hline Organ & Control & EA & TVA & CLA \\
\hline Brain & $1.6 \pm 0.0^{\mathrm{a}}$ & $1.7 \pm 0.1$ & $1.7 \pm 0.1$ & $1.7 \pm 0.0$ \\
\hline Heart & $0.7 \pm 0.0^{\mathrm{b}, \mathrm{a}}$ & $0.6 \pm 0.0^{\mathrm{b}}$ & $0.6 \pm 0.0^{\mathrm{b}}$ & $0.6 \pm 0.0^{\mathrm{a}, \mathrm{b}}$ \\
\hline Kidney & $2.0 \pm 0.1^{\mathrm{a}}$ & $1.6 \pm 0.0^{\mathrm{c}}$ & $1.7 \pm 0.1^{b, c}$ & $1.8 \pm 0.0^{\mathrm{a}, \mathrm{b}}$ \\
\hline Large intestine & $2.4 \pm 0.2$ & $1.9 \pm 0.1$ & $2.5 \pm 0.2$ & $1.9 \pm 0.2$ \\
\hline Liver & $5.8 \pm 0.2^{\mathrm{a}}$ & $4.3 \pm 1.0^{c}$ & $4.9 \pm 0.3^{\mathrm{b}}$ & $5.1 \pm 0.2^{\mathrm{b}}$ \\
\hline Lung & $1.2 \pm 0.1^{\mathrm{a}}$ & $1.0 \pm 0.1^{\mathrm{a}, \mathrm{b}}$ & $0.9 \pm 0.1^{b, c}$ & $1.1 \pm 0.2^{\mathrm{a}, \mathrm{b}}$ \\
\hline Small intestine & $5.5 \pm 0.1$ & $5.2 \pm 0.3$ & $5.4 \pm 0.4$ & $5.3 \pm 0.2$ \\
\hline Spleen & $0.5 \pm 0.0^{\mathrm{a}}$ & $0.2 \pm 0.0^{\mathrm{b}, \mathrm{c}}$ & $0.3 \pm 0.0^{b, c}$ & $0.7 \pm 0.3^{\mathrm{a}}$ \\
\hline Stomach & $4.5 \pm 0.5$ & $3.6 \pm 0.3$ & $3.4 \pm 0.3$ & $3.4 \pm 0.4$ \\
\hline Testis & $0.9 \pm 0.0^{\mathrm{a}}$ & $0.6 \pm 0.1^{\mathrm{c}}$ & $0.8 \pm 0.0^{\mathrm{b}}$ & $0.8 \pm 0.0^{\mathrm{b}}$ \\
\hline
\end{tabular}

Each value represents the mean \pm standard error of the mean $(n=8) .{ }^{a-c}$ Within a row, values with different superscripts are significantly different $(\mathrm{P}<0.05)$. EA, octadecenoic acid; TVA, trans-11 vaccenic acid; CLA, cis-9, trans-11 conjugated linoleic acid.

Table III. Analysis of blood metabolites in mice following repeated oral administration of EA, TVA or CLA for 4 weeks.

\begin{tabular}{lcccc}
\hline Metabolite & Control & EA & TVA & CLA \\
\hline BUN (mg/dl) & $13.9 \pm 3.66^{\mathrm{a}}$ & $15.5 \pm 2.92$ & $21.1 \pm 3.70$ & $14.6 \pm 3.06$ \\
$\mathrm{Ca}(\mathrm{mg} / \mathrm{dl})$ & $6.6 \pm 1.12$ & $5.2 \pm 0.72$ & $6.9 \pm 0.37$ & $7.2 \pm 0.38$ \\
Mg (mg/dl) & $2.7 \pm 0.11^{\mathrm{a}, \mathrm{b}}$ & $2.1 \pm 0.18^{\mathrm{b}}$ & $2.8 \pm 0.25^{\mathrm{a}}$ & $2^{\mathrm{a}}$ \\
Triglyceride (mg/dl) & $49.6 \pm 2.73^{\mathrm{a}}$ & $18.4 \pm 3.61^{\mathrm{b}}$ & $49.6 \pm 17.13^{\mathrm{a}}$ & $61.4 \pm 19.37^{\mathrm{a}}$ \\
Glucose (mg/dl) & $159.4 \pm 8.66^{\mathrm{a}}$ & $126.0 \pm 9.97^{\mathrm{b}}$ & $147.3 \pm 18.99^{\mathrm{a}, \mathrm{b}}$ & $149.9 \pm 18.97^{\mathrm{a}, \mathrm{b}}$ \\
NEFA (ueq/l) & $448.0 \pm 66.78$ & $335.6 \pm 48.04$ & $388.3 \pm 95.66$ & $442.6 \pm 94.62$ \\
Albumin (g/dl) & $2.1 \pm 0.08$ & $2.0 \pm 0.15$ & $2.1 \pm 0.10$ & $2.3 \pm 0.09$ \\
\hline
\end{tabular}

Each value represents the mean \pm standard error of the mean, $n=8 .{ }^{a-c}$ Within a row, values with different superscripts are significantly different $(\mathrm{P}<0.05)$. EA, octadecenoic acid; TVA, trans-11 vaccenic acid; CLA, cis-9, trans-11 conjugated linoleic acid; BUN, blood urea nitrogen; Ca, calcium; Mg, magnesium; NEFA, non-esterified fatty acid.

decreased gradually following treatment with TVA, the reason for this difference remains to be elucidated and requires further investigation.

Ratio of organ/body weight (\%). Significant effects of the three assessed fatty acids on organ weights was observed (Table II). The weights of the liver and testis of mice in the EA, TVA and CLA groups were all lower compared with those in the control groups $(\mathrm{P}<0.05)$. The weights of the spleen, kidney and heart of mice in the EA and TVA groups were also decreased $(\mathrm{P}<0.05$; Table II). However, a previous study reported no gross pathological changes or differences in organ weight following 4 weeks of treatment with TVA (16).

Analysis of blood metabolites. A metabolic profile test (MPT) was initially designed as a pre-symptomatic diagnostic aid, based on the statistical analyses of blood metabolites, to provide an early warning of certain types of metabolic imbalances (17). Previously, it was used to measure physiological characteristics for selection programs designed to improve animal production traits (18). In the present study, 10 blood metabolites were analyzed to determine if any of these factors were correlated with the consumption of pure EA, TVA or CLA. No significant changes were observed in the TVA or CLA group, compared with the control. The EA group exhibited lower levels of $\mathrm{Mg}$ and triglycerides compared with the other groups $(\mathrm{P}<0.05$; Table III). The levels of $\mathrm{Mg}$, triglycerides and glucose were significantly decreased in the EA group compared with those in the control group $(\mathrm{P}<0.05)$.

Circulating levels of cholesterol and the expression of cholesterol 7-hydroxylase. Neither the total cholesterol concentrations or the HDL:total cholesterol ratio in the plasma were significantly different between the groups (Fig. 2A-C). However, the presence of TVA in the diet reduced the levels of plasma LDL cholesterol, compared with the other groups $(\mathrm{P}<0.05$; Fig. 2A-C). In addition, although no significant difference was observed, the expression of CYP7 $\alpha 1$ was increased in the TVA and CLA groups (Fig. 2D). EA is the predominant trans fat in hydrogenated vegetable oils and it has been confirmed that the consumption of EA exerts negative effects on health $(5,19,20)$. In particular, EA increases the activity of cholesterylester transfer protein, which increases the level of VLDL cholesterol and reduces the level of HDL cholesterol (21). CYP7 $\alpha 1$ 

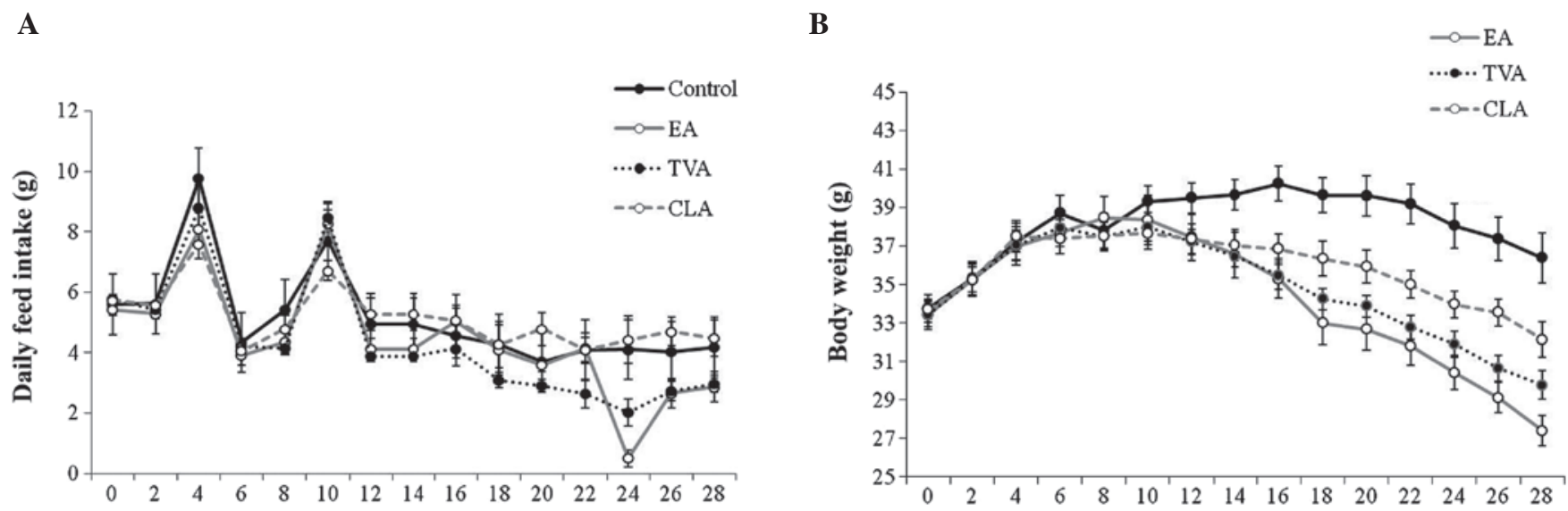

$\mathbf{C}$

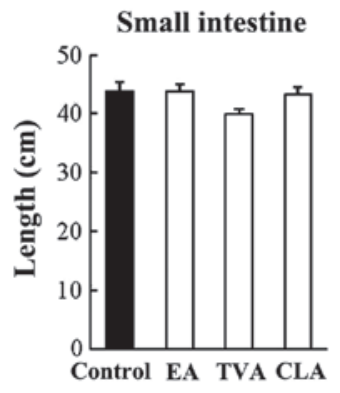

Organ parameters
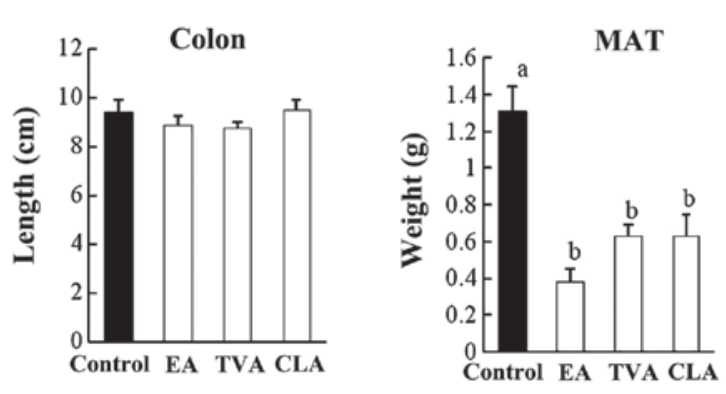

Figure 1. Changes in the (A) daily feed intake $(n=8)$, (B) body weight $(n=8)$ and $(C)$ organ parameters $(n=3)$ of the mice feed EA, TVA or CLA for 4 weeks. Data are expressed as the mean \pm standard error of the mean a and $b \mathrm{P}<0.05$, compared with control group. MAT, mediastinal adipose tissue; EA, octadecenoic acid; TVA, trans-11 vaccenic acid; CLA, cis-9, trans-11 conjugated linoleic acid.

A

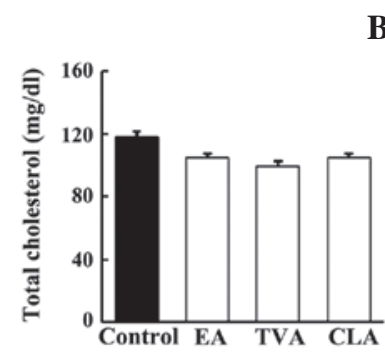

B

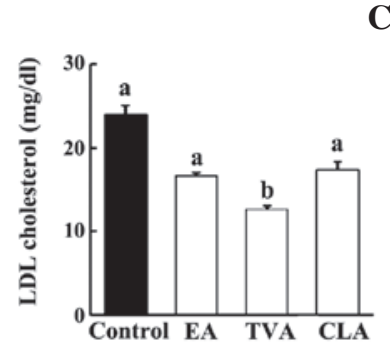

C

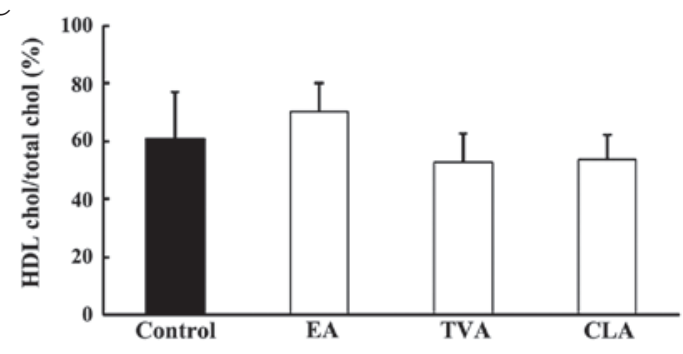

D
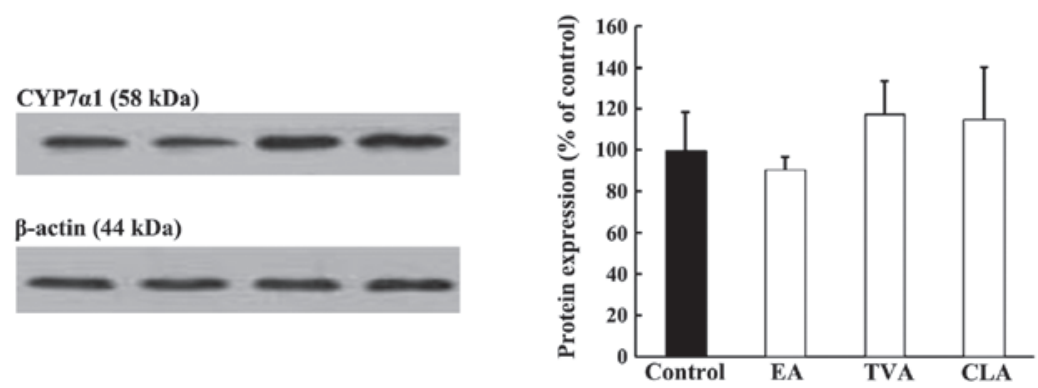

Figure 2. (A-C) Levels of cholesterol and (D) expression levels of in the blood of mice fed EA, TVA or CLA for 4 weeks. The data are expressed as the mean \pm standard error of the mean $(n=8)$. a and $b<<0.05$, compared with control group. EA, octadecenoic acid; TVA, trans-11 vaccenic acid; CLA, cis-9, trans-11 conjugated linoleic acid; CYP7 $\alpha 1$, cholesterol $7 \alpha$-hydroxylase.

is a rate-limiting enzyme in the synthesis of bile acid from cholesterol via the classical pathway (22). A previous study reported primarily neutral effects of TVA/CLA-enriched butter on atherogenic risk factors and reduced aorta fatty streak development $(5,9)$. However, the ratios of atherogenic to anti-atherogenic lipoproteins, VLDL+LDL to HDL, are significantly lower in TVA/CLA-enriched butter groups compared with those of trans-10-C18:1-enriched groups (9). 
A

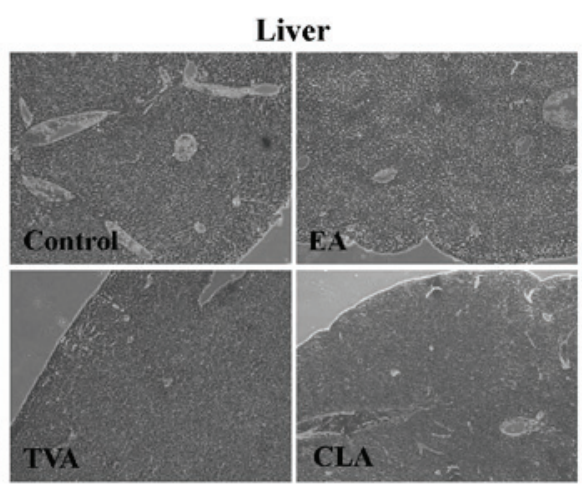

C

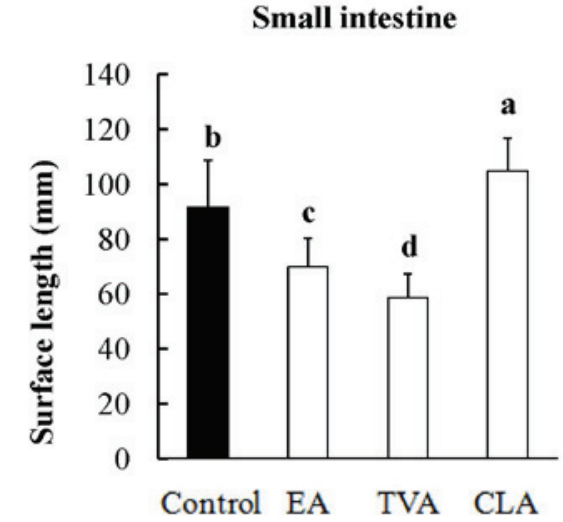

B

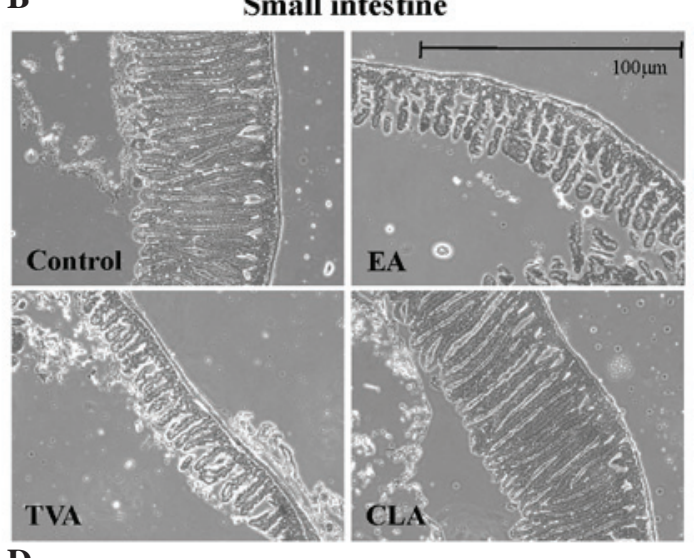

D

TNF-a

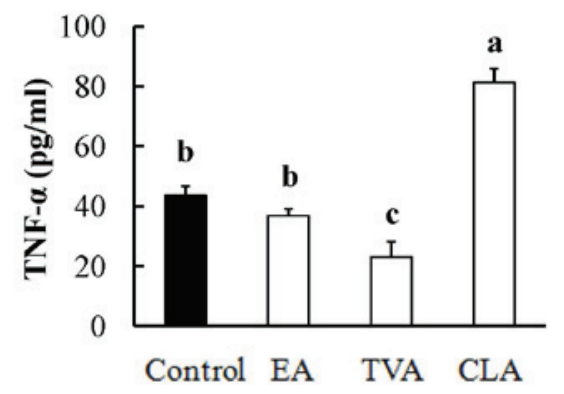

Figure 3. Histochemical analysis of the small intestines and analysis of cytokine production in the plasma of mice fed EA, TVA or CLA for 4 weeks. Representative images of the (A) liver and (B) small intestine (n=80/group; magnification, x100; Olympus CX22). (C) Quantification of the villus length in mice fed EA, TVA or CLA for 4 weeks. (D) Expression levels of TNF- $\alpha(n=8)$ were determined following treatment with EA, TVA or CLA for 4 weeks

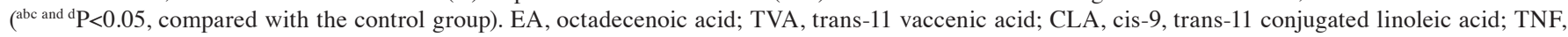
tumor necrosis factor.

Histochemical analysis of the structure of the mouse small intestine. No significant liver injury was observed in any of the groups following 4 weeks of dietary intervention with EA, TVA or CLA (Fig. 3A). However, morphological analyses demonstrated that EA and TVA intervention structurally shortened the length of villi, whereas CLA increased the villi length compared with that of the control (Fig. 3B and C). Villi are finger-like projections in the small intestine, which are important for the digestion and absorption of food (23). These results suggested that EA and TVA had negative effects $(\mathrm{P}<0.05)$ on villi morphogenesis in the small intestines of the mice, where CLA exerted a positive effect $(\mathrm{P}<0.05$; Fig.3D).

Cytokine production in the plasma of mice. Cytokines are fundamental in modulating inflammation, phagocytosis, tissue injury and cell death. Accordingly, the present study measured the plasma levels of circulating TNF- $\alpha$. The CLA group exhibited higher levels of TNF- $\alpha(\mathrm{P}<0.05)$, whereas the TVA group exhibited lower levels, compared with the control group (Fig. 3D). It has been confirmed that the plasma levels of TNF- $\alpha$ are significantly lower in rats fed CLA for 6 weeks compared with control-fed rats $2 \mathrm{~h}$ following LPS challenge, whereas the production of TNF- $\alpha$ in PBS-injection control mice remains unchanged (24). Additionally, a previous report indicated that dietary CLA inhibits the production of TNF- $\alpha$ in weaned pigs challenged with LPS at the protein and mRNA expression levels (25). Previous studies have also indicated that CLA inhibits LPS-stimulated production of TNF- $\alpha$ and expression, partially due to inhibition of the binding activity of nuclear factor- $\mathrm{\kappa B}$. However, the present study did investigate the effects of LPS challenge, therefore, the increased expression of TNF- $\alpha$ by CLA may offer potential in immune-modulation. A pro-inflammatory response can be beneficial for bacterial clearance, however, overactivation of the inflammatory response can lead to cell injury and shock. Therefore, consideration of the appropriate dietary concentrations of CLA is required.

In conclusion, the administration of EA, TVA or CLA in the present study reduced the body weights of the mice, specifically the weights of the liver, testis and MAT. Treatment with EA decreased the levels of magnesium and triglycerides, and the length of the villi, whereas treatment with CLA increased the length of the villi and the expression of TNF- $\alpha$. TVA also reduced the length of the villi, and the levels of LDL and TNF- $\alpha$. Overall, the mice exhibited different responses to EA, TVA and CLA, however, the underlying mechanism remains to be elucidated and requires further investigation.

\section{Acknowledgements}

This study was supported by a grant from the concentrated research professor program for Konkuk University, Seoul, Republic of Korea. 


\section{References}

1. Low JN, Scrimgeour C and Horton P: Elaidic acid (trans-9-octadecenoic acid). Acta Cryst E61: 03730-03732, 2005.

2. Bauman DE, Baumgard LH, Corl BA and Griinari JM: Biosynthesis of conjugated linoleic acid in ruminants. J Anim Sci E77: 1-15, 2000.

3. Wang T and Lee HG: Advances in Research on cis-9, trans-11 Conjugated Linoleic Acid: A Major Functional Conjugated Linoleic Acid Isomer. Crit Rev Food Sci Nutr 55: 720-731, 2015.

4. Wang Y, Lu J, Ruth MR, Goruk SD, Reaney MJ, Glimm DR, Vine DF, Field CJ and Proctor SD: Trans-11 vaccenic acid dietary supplementation induces hypolipidemic effects in JCR: LA-cp rats. J Nutr 138: 2117-2122, 2008.

5. Bassett C, Edel AL, Patenaude AF, McCullough RS Blackwood DP, Chouinard PY, Paquin P, Lamarche B and Pierce GN: Dietary vaccenic acid has antiatherogenic effects in LDLr-/-Mice. J Nutr 140: 18-24, 2010.

6. Dilzer A and Park YH: Implication of conjugated linoleic acid (CLA) in human health. Crit Rev Food Sci Nutr 52: 488-513, 2012.

7. Dupasquier CMC, Patenaude AF, Blackwood DP, Chouinard Y, Lamarche B and Pierce GN: Elaidic and vaccenic TFAs have different effects on atherosclerotic development in low density lipoprotein receptor deficient (LDLr-/-) mice. Ann Nutr Metab 1: 266, 2007.

8. Lock AL, Horne CA, Bauman DE and Salter AM: Butter naturally enriched in conjugated linoleic acid and vaccenic acid alters tissue fatty acids and improves the plasma lipoprotein profile in cholesterol-fed hamsters. J Nutr 135: 1934-1939, 2005.

9. Roy A, Chardigny JM, Bauchart D, Ferlay A, Lorenz S, Durand D, Gruffat D, Faulconnier Y, Sébédio JL and Chilliard Y: Butters rich either in trans-10-C18:1 or in trans-11-C18:1 plus cis-9, trans-11 CLA differentially affect plasma lipids and aortic fatty streak in experimental atherosclerosis in rabbits. Animal 1: 467-476, 2007.

10. Valeille K, Ferezou J, Amsler G, Quignard-Boulange A, Parquet M, Gripois D, Dorovska-Taran V and Martin JCA: cis-9, trans-11-conjugated linoleic acid-rich oil reduces the outcome of atherogenic process in hyperlipidemic hamster. Am J Physiol Renal Physiol 289: H652-H659, 2005.

11. Kanwar RK, Macgibbon AK, Black PN, Kanwar JR, Rowan A Vale $\mathrm{M}$ and Krissansen GW: Bovine milk fat enriched in conjugated linoleic and vaccenic acids attenuates allergic airway disease in mice. Clin Exp Allergy 38: 208-218, 2008.

12. Craig-Schmidt MC: World-wide consumption of trans fatty acids. Atheroscler Suppl 7: 1-4, 2006.
13. Folch J, Lees M and Sloane Stanley GH: A simple method for the isolation and purification of total lipids from animal tissues. J Biol Chem 226: 497-509, 1957.

14. Wang T, Oh JJ, Lim JN, Hong JE, Kim JH, Kim JH, Kang HS, Choi YJ and Lee HG: Effects of lactation stage and individual performance on milk cis-9, trans-11 conjugated linoleic acids content in dairy cows. Asian-Aust J Anim Sci 26: 189-194, 2013.

15. Blewett HJ, Gerdung CA, Ruth MR, Proctor SD and Field CJ: Vaccenic acid favourably alters immune function in obese JCR: LA-cp rats. Br J Nutr 102: 526-536, 2009.

16. Anadon A, Martinez-Larranaga MR, Martinez MA, Ares I, Ramos E, Gomez-Cortes P, Juarez M and de la Fuente MA: 4-Week repeated oral dose toxicity study of dairy fat naturally enriched in vaccenic, rumenic and $\alpha$-linolenic acids in rats. J Agric Food Chem 59: 8036-8046, 2011.

17. Payne JM: The Compton metabolic profile test. Proc R Soc Med 65: 181-183, 1972.

18. Kato Y, Ito $\mathrm{M}$ and Hirooka $\mathrm{H}$ : Genetic parameters of serum vitamin A and total cholesterol concentrations and the genetic relationships with carcass traits in an F1 cross between Japanese Black sires and Holstein dams. J Anim Sci 89: 951-958, 2011.

19. Meijer GW, Tol AV, Berkel TV and Weststrate JA: Effect of dietary elaidic versus vaccenic acid on blood and liver lipids in the hamster. Atherosclerosis 157: 31-40, 2001.

20. Fournier N, Attia N, Rousseau-Ralliard D, Vedie B, Destaillats F Grynberg A and Paul JL: Deleterious impact of elaidic fatty acid on ABCA1-mediated cholesterol efflux from mouse and human macrophages. Biochim Biophys Acta 182: 303-312, 2012.

21. Abbey M and Nestel PJ: Plasma cholesteryl ester transfer protein activity is increased when trans-elaidic acid is substituted for cis-oleic acid in the diet. Atherosclerosis 106: 99-107, 1994.

22. Jelinek DF, Andersson S, Slaughter CA and Russell DW: Cloning and regulation of cholesterol 7 alpha-hydroxylase, the rate-limiting enzyme in bile acid biosynthesis. J Biol Chem 265: 8190-8197, 1990.

23. Ryan G. Spurrier and Tracy C. Grikscheit: Tissue Engineering the Small Intestine. Clin Gastroenterol Hepatol 11: 354-358, 2013.

24. Yang M and Cook ME: Dietary conjugated linoleic acid decreased cachexia, macrophage tumor necrosis factor- $\alpha$ production and modifies splenocyte cytokines production. Exp Biol Med (Maywood) 228: 51-58, 2003.

25. Zhao L, Yin J, Li D, Lai C, Chen X and Ma D: Conjugated linoleic acid can prevent tumor necrosis factor gene expression by inhibiting nuclear factor binding activity in peripheral blood mononuclear cells from weaned pigs challenged with lipopolysaccharide. Arch Anim Nutr 59: 429-438, 2005. 